\title{
Epidemiología de la enfermedad inflamatoria intestinal crónica en cinco áreas de Asturias. España
}

\author{
C. SARO GISMERA, S. RIESTRA MENÉNDEZ', R. SÁNCHEZ FERNÁNDEZ², \\ A. MILLA CRESPO ${ }^{2}$, M. LACORT FERNÁNDEZ, G. ARGÜELLES FERNÁNDEZ, \\ Z. CHOBAK ${ }^{3}$, J. I. FLORIDO MANCHEÑO ${ }^{4}$, J. L. ANTÓN MAGARZO, \\ A. ALTADILL ARREGUI ${ }^{5}$, F. VIZOSO ${ }^{5}$, E. PINEDA GARCÍA ${ }^{2}$ \\ E. FERNÁNDEZ DE OCARIZ ARCHS ${ }^{4}$, J. ALBERT COLOMER ${ }^{7}$, J. GARCÍA PÉREZ7 ${ }^{7}$ \\ L. LÓPEZ RIVAS ${ }^{6}$, J. L. S. LOMBRAÑA ${ }^{7}$
}

Hospital de Cabueñes. ${ }^{1}$ Hospital Valle del Nalón. ${ }^{2}$ Hospital Alvarez Buylla. ${ }^{3}$ Hospital de Jarrio. ${ }^{4}$ Hospital Severo Ochoa. ${ }^{5} \mathrm{Hospital}$ de Jove. ${ }^{6} \mathrm{Hospital}$ San Agustín. ${ }^{7} \mathrm{Hospital}$ Central. Asturias

EPIDEMIOLOGY IN INFLAMMATORY BOWEL DISEASE IN FIVE AREAS OF ASTURIAS. SPAIN

\section{RESUMEN}

Objetivo: La epidemiología de la enfermedad inflamatoria intestinal crónica (EIIC) es una poderosa herramienta de investigación que contribuye a la evaluación de los factores medioambientales que influyen en su etiología. El objetivo de este estudio es conocer distintos aspectos epidemiológicos de la EIIC en nuestro medio.

Pacientes y métodos: Estudio epidemiológico descriptivo, poblacional, multicentrico, retrospectivo entre 1954 y 1993 y prospectivo entre 1994 y 1997. Se incluyen 1018 enfermos mayores de 14 años, diagnosticados de EIIC en 5 áreas del Principado de Asturias (España), con un censo de 461.965 habitantes.

Resultados: Del total de 1018 identificados [565 CU (55,5\%) (incluyendo proctitis), 415 EC $(40,8 \%)$ y 38 CI $(3,7 \%)], 482$ son mujeres $(47,2 \%)$ y 536 varones $(52,8 \%)$, con una relación $\mathrm{V} / \mathrm{M}$ de 1,11 . La edad media al diagnóstico es de 39,49 $\pm 1,08$ (IC; 95\%: 38,41 - 40,57), [CU: $43,95 \pm 1,47$; EC: $33,53 \pm 1,51$; CI: $38,26 \pm 5,14] . p=0,000$. La edad media de inicio de síntomas previo al diagnóstico es 37,66 $\pm 0,97$ (CU: $42,84 \pm 1,34$; EC: $30,68 \pm 1,40$; CI: 36,74 $\pm 4,86$ ( $\mathrm{p}=0,000$ ). El diagnóstico de CU ha sido posible con criterios clínicos en el 97,34\% ( $p=n s)$, criterios endoscópicos en el 96,63\% ( $p=0,000)$ y criterios histológicos en el $90,26 \%(p=0,000)$. En la EC: criterios radiológicos 83,61\% ( $p=$ $0,000)$. El nivel cultural es superior en la EC: $57,57(p=0,0005)$. Asociación familiar del 8,4\%. Extensión: en la CU: proctitis 13,6\%, 26,9\% colitis distal, $26 \%$ colitis izquierda, $6 \%$ colitis extensa y el $20 \%$ pancolitis; En la EC el 30,3\% tienen afectación de íleon terminal, el 16,7\% colon, el $41,3 \%$ colon e intestino, el $11,7 \%$ son intestinales extensas y el $3,7 \%$ tienen afectación gastro-duodenal; En la CI destaca un 39,5\% de afectación discontinua. La media de cirugías necesarias para el control de la enfermedad es de $0,44 \pm 6,11,(26,62 \%$ de los enfermos $)$. CU: $0,12 \pm$ $3,33(9,91 \%)$; EC: $0,91 \pm 12,9(50,36 \%), p=0,000$. Tasa de Mortalidad de 47,15 /1000 habitantes (CU: $\mathrm{T}=61,94 ; \mathrm{EC}: \mathrm{T}=26,50 ; \mathrm{CI}: \mathrm{T}=0,004)$ $\mathrm{p}=0,046$. RMS: 0,467 (CU: 6,14; EC: 2,63; CI: 100)

Conclusiones: Este estudio que abarca una importante población de enfermos, pretende aportar nuestros resultados epidemiológicos a la Enfermedad Inflamatoria Intestinal Crónica. Nuestros resultados no difieren substancialmente de los de otras publicaciones. La colitis ulcerosa y la enfermedad de Crohn así como el sexo, se distribuyen uniformemente. La elevada asociación familiar entre estas enfermedades sugiere un origen genético de la EIIC. La enfermedad de Crohn se expresa con mayor morbilidad reflejada en los requerimientos quirúrgicos, pero sin embargo con menor mortalidad que en la colitis ulcerosa.

PALABRAS CLAVE: Enfermedad Inflamatoria Intestinal Crónica. Colitis ulcerosa. Enfermedad de Crohn. Colitis indeterminada. Epidemiología.

\section{ABSTRACT}

Aims: The epidemiologic analysis inflammatory bowel disease (IBD) is a powerful research tool to assess the contribution of environmental factors to its etiology. IBD has been reported to have varying frequencies in different parts of the world, and there seem to be significant differences in the disease pattern and clinical course. The aim of the present study was to assess the disease pattern of IBD in Asturias (Spain).

Patients and methods: A descriptive epidemiological population based study, retrospective (1954-1993) and prospective (1994-97), was performed to study 1018 patients found, bigger than 14 years, to have $I B D$, in five areas of Asturias (Spain) (461.965 inhabitants).

Results: During the period of time studied, we diagnosed 1018 IBD [565 ulcerative colitis (55.5\%), 415 (40.8\%) Crohn's disease and 38 $(3.7 \%)$ indeterminate colitis], with 482 females $(47.2 \%), 536$ males (52.8\%), and male/female: 1.11. Age at diagnosis were $39.49 \pm 1.08$ (95\% CI : 38.41 - 40.57); (UC: $43.95 \pm 1.47$; CD: $33.53 \pm 1.51$; IC: $38.26 \pm$ 5.14. $p=0.000$. Age at onset previously at diagnosis for $U C$ : $42.84 \pm 1.34$; $C D: 30.68 \pm 1.40$; IC: $36.74 \pm 4.86(p=0.000)$. Diagnosis criteria: UC: syntomatic $97.34 \%(p=n s)$, endoscopy $96.63 \%(p=$ $0.000)$, pathology $90.26 \%(p=0.000) . C D$ : radiology $83.61 \%(p=$ $0.000)$. Study level in CD: $57.57(p=0.0005)$. Family history: $8.4 \%$. The most frequent involvement at diagnosis of UC was proctitis only, in $13.6 \%, 26.9 \%$ rectum and sigmoid, $26 \%$ left colitis, $20 \%$ pancolitis, and in CD colon only, in 16.7\%, 30.3\% terminal ileum, $41.3 \%$ ileo-colon of the patients. This also helps to explain the differences in severity, need for surgery, and survival noted between community based studies.

Conclusions: We highlight the uniformity of distribution of the inflammatory bowel disease in relation to types and sex. The high frequency of familial Crohn's disease suggests a genetic predisposition Highlighting a bigger morbilidad for the Crohn's Disease reflected in the surgical requirements, but however with smaller mortality that in ulcerative colitis.

KEY WORDS: Inflammatory Bowel Disease. Ulcerative Colitis. Crohn's Disease. Indeterminate colitis. Epidemiology.

Trabajo aceptado: 17 de enero de 2003

Correspondencia: Cristina Saro Gismera. S. Ap. Digestivo. Hospital de Cabueñes.Gijón. Asturias. Camino de Cabueñes S/N. 33394. Tfno: 985.185000 . Fax: 985.367169. e-mail: csarog@meditex.es 
C. Saro Gismera, S. Riestra Menéndez, R. Sánchez Fernández, A. Milla Crespo, M. Lacort Fernández, G. Argüelles Fernández, Z. Chovac, J. I. Florido Mancheño, J. L. Antón Magarzo, A. Altadill Arregui6, F. Vizoso, E. Pineda García, E. Fernández de Ocariz Archs, J. Albert Colomer, J. García Pérez, L. López Rivas, J. L. S. Lombraña. Epidemiología de la enfermedad inflamatoria intestinal crónica en cinco áreas de Asturias. España. An Med Interna (Madrid) 2003; 20: 232-238.

\section{INTRODUCCIÓN}

Colitis ulcerosa (CU), enfermedad de Crohn (EC) y Colitis Indeterminada (CI), se agrupan con el termino de Enfermedad Inflamatoria Intestinal Crónica (EIIC), habiendo sido clásicamente bien definidas en sus aspectos clínicos y estructurales desde los primeros trabajos de Evans y Acheson (1), y desde 1964 también en la CI (2). Se caracterizan por períodos recurrentes de inflamación y destrucción del tejido, con gran variación en la evolución de estos períodos en los distintos sujetos. Esta variabilidad del curso clínico se influencia por la genética, factores medioambientales y el sistema inmunológico. A pesar de las recientes contribuciones, los adelantos en la ingeniería genética y la biología molecular, continúan presentando sin embargo, numerosos interrogantes en relación con su etiopatogenia. Entendemos que a través de los resultados de los estudios epidemiológicos, se llega al diseño de estudios científicos que permiten averiguar finalmente el origen de la enfermedad y por tanto conseguir un tratamiento eficaz. Con el presente Estudio Epidemiológico, pretendemos conocer la distribución de la Enfermedad Inflamatoria Intestinal Crónica y su variabilidad en nuestro medio.

\section{PACIENTES Y MÉTODOS}

Tipo de estudio: estudio epidemiológico, descriptivo, poblacional y multicentrico.

Periodo de tiempo: retrospectivo desde 1954 hasta 31 de Diciembre de 1993 y prospectivo desde el 1 de enero de 1994 hasta el 31 de diciembre de 1997.

Criterios de inclusión: se han incluido en este estudio el conjunto de los 1018 enfermos (565 CU, $415 \mathrm{EC}$ y $38 \mathrm{CI}$ ) y que componen la muestra total de pacientes diagnosticados de EIIC en las 5 áreas de Asturias participantes, tanto en régimen hospitalario como en consultas externas, que cumplen los criterios diagnósticos descritos por Lennard-Jones y Truelove (3-5) para EC y CU respectivamente. Para la CI hemos empleado los criterios descritos por Ashley B. Price (2). Se han excluido causas infecciosas y otras causas reconocidas de inflamación. Todos ellos han alcanzado edad mayor de 14 años al cierre del estudio. Incluimos vivos y fallecidos; intervenidos o no, incluyendo las CU con Panproctocolectomía. Todos se controlan en nuestra área y las evaluaciones se refieren al ultimo control realizado. El estudio ha sido realizado en forma prospectiva en los últimos 4 años y el resto en forma retrospectiva. Los resultados obtenidos con cada uno de estos grupos han sido similares en sus aspectos más relevantes, por lo que son expuestos en su conjunto.

Población: el Principado de Asturias, autonomía situada al norte de España, está dividida en 8 Áreas Sanitarias, con una población adulta de 931.097 habitantes. Nuestro estudio se ha realizado en 5 de estas áreas (I,II,V,VII y VIII), con una población adulta de 461.965 habitantes $(49.61 \%$ de los habitantes de Asturias). $48,13 \%$ varones y $51,86 \%$ de mujeres. Tiene una climatología oceánica, templada y húmeda (precipitaciones de
800-1500 mm) sin grandes cambios estacionales. Consta de un centro industrializado $(28,95 \%)$ y altamente urbanizado, con población minera asentada en yacimientos carboníferos (Nalón, Turón y Aller), distribuyéndose en forma dispersa la agricultura $(13,69 \%)$, y la pesca $(0,92 \%)$. El sector terciario de Servicios representa el $56,44 \%$ de la población.

Metodología: los pacientes han sido identificados en el estudio retrospectivo, revisando los archivos generales del hospital, los archivos de endoscopia, anatomía patológica y consultas externas, y en el periodo prospectivo mediante detección de los casos incidentes, como se refiere en nuestro estudio de incidencia y prevalencia. Hemos utilizado un mismo cuestionario para todos los enfermos, con recogida de los datos objeto del estudio a través de la historia clínica y/o mediante encuesta directa con el enfermo. Todos los casos han sido revisados por el investigador principal de cada área, habiendo contactado y mantenido reuniones periódicas con los médicos gastroenterólogos y cirujanos del área, tanto públicos como privados. Se han incluido y cruzado los casos recogidos en una base de datos (Dbase IV), con el fin de evitar la duplicidad de los pacientes. En todos los enfermos se ha realizado seguimiento clínico hasta el cierre del estudio. Se ha calculado el nivel cultural a través del nivel de estudios, tipificando 5 niveles $(\mathrm{A}=\sin$ estudios; $\mathrm{B}=$ estudios primarios; $\mathrm{C}=$ estudios secundarios; $\mathrm{D}=$ diplomados; $\mathrm{E}=$ estudios superiores). La extensión aplicada es aquella que corresponde al estudio más reciente, antes de la primera intervención quirúrgica. En la CU se aplican las siguientes extensiones: proctitis como afectación de $15 \mathrm{~cm}$ proximales al margen anal, colitis distal como afectación de recto y sigma, colitis izquierda como afectación de sigma y colon descendente con o sin afectación rectal, colitis extensa como afectación hasta el ángulo hepático y pancolitis como afectación de todo el colon. Hemos utilizado para la EC, la siguiente extensión: Colon, Íleon terminal, Colon + íleon (incluimos afectación de Ciego y Colon derecho), Intestinal extensa y Gastro-duodenal. En los requerimientos quirúrgicos, incluimos cirugía menor por fístulas y abscesos perianales. En el estudio de mortalidad, incluimos las muertes por la propia enfermedad y por otras causas. Se han analizado los resultados de los dos grupos de población, retrospectivo y prospectivo, sin encontrar diferencias significativas (excepto en el número de cirugías), por lo que los resultados expuestos corresponden al conjunto de ambos grupos. Se han analizado los resultados de los dos grupos de población, retrospectivo y prospectivo, sin encontrar diferencias significativas (excepto en el número de cirugías), por lo que los resultados expuestos corresponden al conjunto de ambos grupos.

Analizamos: frecuencia y comparación por sexos; se compara la edad al diagnóstico de las tres entidades, así como el periodo de tiempo sintomático previo al diagnóstico, de lo que inferimos la edad media de inicio real de las distintas enfermedades.

Evaluamos cuáles han sido la frecuencia de positividad de las pruebas diagnósticas realizadas (clínicos, radiológicos, endoscópicos e histológicos) en cada una de las enfermedades, dando como positivo aquellos criterios descritos por Lennard-Jones, Truelove y Ashley B. Price (2-5), calculando el 
porcentaje con positividad diagnóstica y comparando cada criterio en las tres enfermedades que estudiamos. Igualmente se compara el nivel cultural; porcentajes de asociación familiar, comparando las tres enfermedades; severidad de la enfermedad en relación con su extensión; número y frecuencia de enfermos operados (porcentaje), número de cirugías practicadas, media de cirugías en relación a la totalidad de enfermos y número de cirugías por enfermo operado (TQ = Tasa Quirúrgica: $\mathrm{n}^{\circ}$ de Cirugías $/ \mathrm{n}^{\circ}$ Enfermos operados); exponemos el número de enfermos fallecidos, frecuencia, tasas de mortalidad, comparando estas entre las tres enfermedades y con la mortalidad de la población general en nuestro medio.

Método estadístico: para la comparación entre variables cualitativas, se ha empleado la prueba de la $\chi 2$ y el análisis de varianza para comparar variables cuantitativas. Los intervalos de confianza (95\%) de las tasas, se han calculado mediante las distribuciones binomial y de Poisson. La tasa de mortalidad se expresa en número de muertes por 1000 habitantes. Hemos utilizado las Tablas de Mortalidad General de Asturias publicadas por la Consejería de Sanidad en 1994, para establecer las comparaciones. Se han comparado las muertes observadas con las esperadas para obtener las Ratios de Mortalidad Stándar (R.M.S.)

\section{RESULTADOS}

El número de enfermos que cumplen los criterios de inclusión de EIIC en el área estudiada ha sido de 1018; 565 CU $(55,5 \%), 415$ EC $(40,8 \%)(p=n s)$, y 38 CI $(3,7 \%)$. De los 1018 pacientes, 482 son mujeres $(47,2 \%), 536$ varones $(52,8 \%), \mathrm{p}=$ ns. Observamos una relación varón/mujer de 1,11, (CU: 1,08; EC: 1,15; CI: 1,11) (Fig. 1).

Edad al diagnóstico. La media de edad de diagnóstico de la EIIC es de $39.49 \pm 1.08$ (IC; $95 \%: 38.41$ - 40.57), en la CU: 43.95 " 1.47 (IC; $95 \%: 42,48$ - 45,42), en la EC: $33,53 \pm 1,51$ (IC; 95\%: 32,02 - 35,04), y en la CI: 38,26 \pm 5,14 (IC; 95\%: $33,12-43,4), p=0,000$.

El periodo de tiempo sintomático previo al diagnóstico que denominamos periodo de latencia de la enfermedad (EIIC $=1,83 \pm 0,29$ años), está significativamente aumentado en la EC $(2,85 \pm 0,54$ años) en relación a la CU $(1,11 \pm 0,23$ años) $(\mathrm{p}=0,000)$, y por ello la edad de inicio de los síntomas

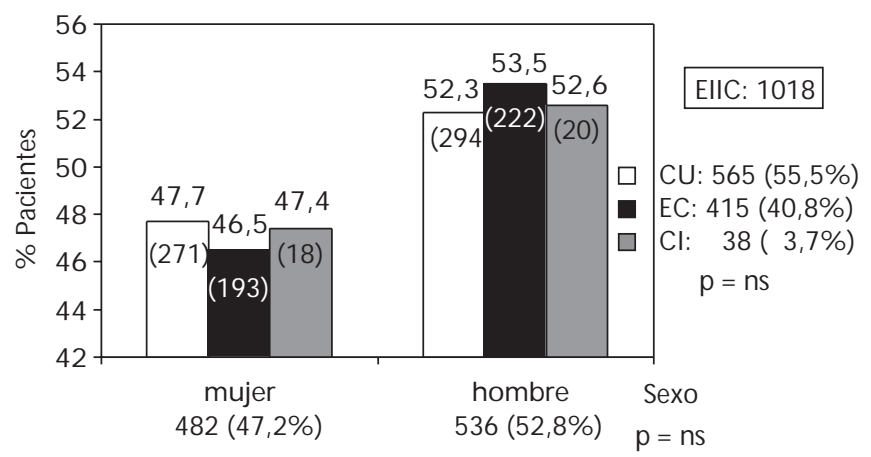

Fig. 1. Frecuencia. Sexo. ElIC: enfermedad inflamatoria intestinal crónica; CU: colitis ulcerosa; EC: enfermedad de Crohn; $\mathrm{Cl}$ : colitis indeterminada. Distribución por sexo. $p=$ N.S. Distribución por diagnósticos. $p=$ N.S.
(EIIC $=37,66 \pm 0,97$ años), es significativamente más precoz en la EC $(30,68 \pm 1.40$ años) que en la CU $(42,84 \pm 1,34$ años $)$ $(\mathrm{p}=0,000)$. En nuestro grupo de enfermos con CI, el periodo de latencia $(1,52 \pm 3,32$ años $)$ y la edad de inicio sintomático (36,74 \pm 4,86 años), se encuentra en situación intermedia.

Criterios diagnósticos. Analizamos las Técnicas que han permitido el diagnóstico. Los pacientes que cumplen Criterio clínico son: 550 en CU (97,34\%), 396 en EC (95,42\%), 35 en CI $(92,10 \%)(\mathrm{p}=\mathrm{ns})$; Criterio endoscópico: en $546 \mathrm{CU}(96,63 \%)$, en 197 EC (47,46\%), en 37 CI (97,36\%), p = 0,000; Criterio histológico de CU en 510 casos $(90,26 \%)$, de EC en 252 casos $(60,72 \%)$ y de CI en 33 casos $(86,84 \%), p=0,000$; Criterio Radiológico (Rx. baritado) de CU en 235 casos $(41,59 \%)$, de EC en 347 casos $(83,61 \%)$ y de CI en 14 casos $(36,84 \%), p=0,000$.

Nivel cultural. Como puede observarse en la tabla I, los pacientes con EC presentan un nivel de estudios superior al de los pacientes con $\mathrm{CU}$, con una significación estadística de $\mathrm{p}=$ 0,0005 .

Asociación familiar. El 8,4\% de los enfermos tienen familiares en 1er o 21 grado con EIIC. La CU presenta un nivel de asociación del 7,6\%, mientras que en la EC la asociación es del 8,6\% ( $\mathrm{p}=0,053)$. En la CI observamos historia familiar en el $18,9 \%$ ( $\mathrm{p}=\mathrm{ns})$ (Fig. 2).

Extensión. Los datos referentes a la extensión, se observan en la tabla II, destacando un $13,6 \%$ de proctitis ulcerosa, seguido de un 26,9\% de colitis distales. En la CI destaca un 39,5\% con localización discontinua. El 30,3\% de los pacientes con EC tienen afectación de íleon terminal, el 16,7\% sólo colon, un $41.3 \%$ se localiza en colon e intestino, el $11,7 \%$ son intestinales extensas y el 3,7\% tienen afectación gastro-duodenal.

Cirugía. (Tabla III). La media de cirugías necesarias para el control de la EIIC ha sido de 0,44 $\pm 6,11$, (corresponde al $26.62 \%$ de los enfermos). La EC precisó una media de 0,91 \pm 12,9 cirugías $(50,36 \%)$ con una significación de $\mathrm{p}=0,000$ en relación con CU y CI.

Mortalidad. (Tablas IV y V). Observamos una tasa de mortalidad de 47,15, superior en CU $(T=61,94 ; p=0,046)$, sin diferencias en relación con el sexo. La RMS que puede observarse en la tabla $\mathrm{V}$, implica un aumento del riesgo relativo de muerte, tanto para el conjunto de los pacientes con EIIC, como para CU y EC, con relación a la mortalidad de la población general.

\section{DISCUSIÓN}

En una primera parte de este trabajo, expresábamos las tasas de incidencia y prevalencia en 5 áreas del Principado de Asturias. Ahora, pretendemos ampliar la información con otros datos epidemiológicos. Los resultados de los estudios epidemiológicos sobre la EIIC publicados hasta la actualidad, a menudo son dispares, como expresábamos en un estudio previo de nuestro grupo (6). El esfuerzo, por lo tanto, debe encaminarse en conseguir uniformidad metodológica, que permita llegar a conclusiones univocas.

$\mathrm{Al}$ igual que en estudios previos españoles (7-10), no hemos encontrado diferencias en la distribución entre CU y EC, así como tampoco encontramos diferencia en ninguno de los grupos de enfermedad en relación al sexo. Los dos estudios más recientes presentan unos resultados con predominio de mujeres en la EC en el Estudio Cooperativo Europeo (11), mientras que en el estudio de Brullet y cols. (1,4/1,1) (12), se muestra predominio 
TABLA I

NIVEL CULTURAL

\begin{tabular}{|c|c|c|c|c|c|c|c|c|c|c|}
\hline & A. & $\%$ & B. & $\%$ & C. & $\%$ & D. & $\%$ & E. & $\%$ \\
\hline $\begin{array}{l}\text { EllC } \\
(956)\end{array}$ & 9 & $(0,9)$ & 549 & $(57,4)$ & 221 & $(23,1)$ & 111 & $(11,6)$ & 66 & $(6,9)$ \\
\hline $\begin{array}{l}\text { CU } \\
\text { (523) }\end{array}$ & 8 & $(1,52)$ & 337 & $(64,43)$ & 108 & $(20,65)$ & 47 & $(8,98)$ & 23 & $(4,39)$ \\
\hline $\begin{array}{l}\text { EC* } \\
\text { (396) }\end{array}$ & 1 & $(0,25)$ & 198 & (50) & 101 & $(25,50)$ & 58 & $(14,64)$ & 38 & $(9,59)$ \\
\hline $\begin{array}{l}\mathrm{Cl} \\
(37)\end{array}$ & & & 14 & $(37,83)$ & 12 & $(32,43)$ & 6 & $(16,21)$ & 5 & $(13,51)$ \\
\hline
\end{tabular}

EllC: enfermedad inflamatoria intestinal crónica; CU: colitis ulcerosa; EC: enfermedad de Crohn; Cl: colitis indeterminada, Nivel de estudios $(*) p=0,0005$.

( $A=\sin$ estudios; $B=$ estudios primarios; $C=$ estudios secundarios; $D=$ diplomados; $E=$ estudios superiores $)$.

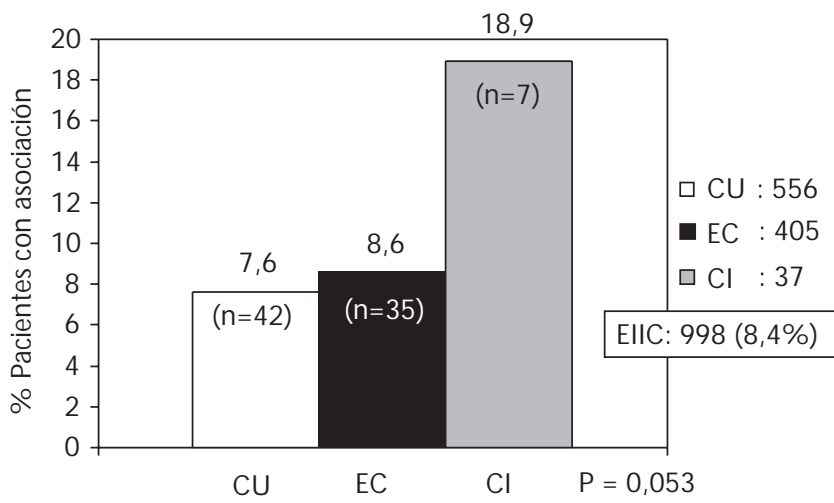

Fig. 2. Asociación Familiar. ElIC: enfermedad inflamatoria intestinal crónica; CU: colitis ulcerosa; EC: enfermedad de Crohn; $\mathrm{Cl}$ : colitis indeterminada. Relación de asociación familiar entre EC y CU: $p=$ 0,053 .

de varones para esta misma enfermedad. En la CU ambos resultados son uniformes, con predominio de varones. En ambos casos sin significación estadística. En el estudio de Gower y cols. (13), las mujeres tienen más tendencia a padecer EC y menor a padecer CU. El resto de los autores, obtienen resultados dispares (14-19). Podemos por lo tanto deducir que no existe uniformidad en la distribución del sexo en este grupo de enfermedades y que probablemente su presentación sea aleatoria.

Edad al diagnóstico. En el consenso de Viena 1998 (20), se valora exclusivamente la edad al diagnóstico como variable fácilmente reproductible y por su relevancia social y clínica. Nuestro grupo se ha interesado además por la edad de inicio sintomático a fin de intentar discriminar a través de ésta variable, el diagnóstico definitivo de la CI. Sin embargo, no hemos encontrado diferencias significativas que nos permitan la discriminación, ya que la CI presenta un periodo de latencia y tiempo de inicio intermedio entre CU y EC. Encontramos, al igual que otros autores $(15,18,21-23)$, un tiempo de latencia muy superior en la EC sobre la CU, lo que parece sugerir que este grupo de enfermos presenta una sintomatología más larvada, que hace retrasar el diagnóstico.

La edad media al diagnóstico, significativamente diferente entre $\mathrm{CU}$ y EC, es similar a la de otras series publicadas
(7,10,19,24-26). Monferrer y cols. 26 refiere una edad media al diagnostico de 38,6 años para la CI, (igual a la nuestra) y Stewenius y cols. (21) encuentra un pico de máxima incidencia en las mujeres entre 20 y 29 años, mientras que en los varones este pico se encuentra entre los 10 y 19 años.

Criterios diagnósticos. Destacamos la baja sensibilidad discriminativa de la histología en nuestro medio, siendo la sintomatología y la técnica endoscópica más sensibles para la $\mathrm{CU}$, como ya ha sido descrito por Pera y cols. (27), autor que señala un $90 \%$ de seguridad diagnóstica. Por el contrario, en la EC, es la Radiología la técnica de mayor seguridad diagnóstica, aunque este dato está sesgado debido a la alta frecuencia de enfermos que sólo tienen localización ileal de su enfermedad y por tanto radiológicamente demostrable. No podemos comparar con los resultados del Estudio Cooperativo Europeo (11), dado que en ese trabajo evalúan solo la técnica empleada pero no especifican sobre los resultados de la misma. Tampoco hemos incluido otras técnicas de imagen tales como ecografía, TAC, o estudios isotópicos, dado que por el largo periodo de tiempo analizado, esto no sería factible en los pacientes de diagnóstico más antiguo.

Obsevamos un nivel cultural superior en la EC y en la CI en relación con la $\mathrm{CU}$, aunque ésta última no en forma significativa, quizás por el pequeño tamaño de la muestra. Nuestros datos globales no difieren de los reflejados en otras publicaciones $(17,28)$, pero sí destacamos la diferencia existente entre las distintas enfermedades que componen la EIIC, a diferencia de los resultados del estudio de Van Gossum y cols. (29).

Asociación familiar. Las diferencias étnicas están empezando a desaparecer según nos aseguran Cottone y cols. (30), subrayando el papel de los factores medioambientales en la génesis de la enfermedad. Señalan que los parientes en primer grado de pacientes con EC tienen un riesgo relativo de enfermedad entre 2 y 4 . El factor de riesgo actualmente reconocido más fuerte para la enfermedad de Crohn es tener un pariente con la enfermedad. Satsangi J y cols. (31) aseguran que la concordancia entre los pares de gemelos y hermanos proporciona fuerte evidencia de que los factores genéticos son importantes en la patogénesis de la enfermedad.

En la actualidad, la hipótesis más fiable es que la EIIC tiene una patogénesis multifactorial: antígenos y factores medioambientales que actúan en los pacientes genéticamente predispuestos. Todos los estudios de agregación familiar indican una alta 
TABLA II

EXTENSIÓN (1.080 CASOS)

\begin{tabular}{lccccccc}
\hline Extensión & CU & $(\%)$ & $\mathrm{Cl}$ & $(\%)$ & Extensión & EC & $(\%)$ \\
\hline Proctitis & 77 & $(13,6)$ & 2 & $(5,2)$ & Íleon & 126 & $(30,3)$ \\
Distal & 152 & $(26,9)$ & 6 & $(15,8)$ & Intestinal & 46 & $(11,7)$ \\
C. Izq. & 147 & $(26,0)$ & 7 & $(18,4)$ & Colon & 70 & $(16,7)$ \\
Extensa & 34 & $(6,0)$ & 3 & $(7,9)$ & Colon+lntestinal & 174 & $(41,3)$ \\
Pancolitis & 113 & $(20)$ & 5 & $(13,2)$ & & & \\
Discontinuo & 42 & $(7,5)$ & 15 & $(39,5)$ & E.G.D. & 16 & $(3,7)$ \\
\hline
\end{tabular}

ElIC: enfermedad inflamatoria intestinal crónica; CU: colitis ulcerosa; EC: enfermedad de Crohn; Cl: colitis indeterminada.

TABLA III

REQ UERIM IENTOS Q UIRÚRGICOS

\begin{tabular}{lccccc}
\hline & \multicolumn{2}{c}{ № de enfermos operados $(\%)$} & M edia (DE) & Cirugías & TQ \\
\hline EIIC & 271 & $(26,62)$ & $0,44 \pm 6,11$ & 453 & 1,67 \\
CU & 56 & $(9,91)$ & $0,12 \pm 3,33$ & 69 & 1,23 \\
EC ${ }^{*}$ & 209 & $(50,36)$ & $0,91 \pm 12,9$ & 277 & 1,8 \\
Cl & 6 & $(15,78)$ & $0,18 \pm 14,5$ & 7 & 1,17 \\
\hline
\end{tabular}

D E: Desviación Estándar; Tasa Q uirúrgica (TQ ) = Razón: n1 de Cirugías / Enfermos operados.

EIIC: enfermedad inflamatoria intestinal crónica; CU: colitis ulcerosa; EC: enfermedad de Crohn; Cl: colitis indeterminada, Cirugías $(*) ; p=0,000$.

TABLA IV

MORTALIDAD EN EIIC

\begin{tabular}{lcccc}
\hline & $\mathrm{CU}$ & $\mathrm{EC}$ & $\mathrm{Cl}$ & EllC \\
\hline Total & 35 & 11 & 2 & 48 \\
& $(6,19 \%)$ & $(2,65 \%)$ & $(5,26)$ & $(4,71 \%)$ \\
M ujeres & $(\mathrm{T}=61,94)$ & $(\mathrm{T}=26,50)$ & $(\mathrm{T}=0,004)$ & $(\mathrm{T}=47,15)$ \\
& $(5,53 \%)$ & $(2,07 \%)$ & 1 & $(5,55 \%)$ \\
Hombres & 20 & 7 & 1 & $(4,14 \%)$ \\
& $(6,8 \%)$ & $(3,15 \%)$ & $(5 \%)$ & 28 \\
& & & $(5,22 \%)$ \\
\hline
\end{tabular}

$\mathrm{T}=$ Tasa de mortalidad (№ muertes/ 1000 habitantes).

M ortalidad comparada: CU/ EC: $p=0,046$.

M ortalidad comparada: Sexos: $\mathrm{p}=\mathrm{NS}$.

incidencia de familiares con ambas patologías, pero sobretodo en la EC. En los últimos años, se ha publicado que la agregación familiar es mucho más alta en EC (entre un 4,5 y 18,8\%) que en CU. El estudio Spanish Epidemiological and Economic Study Group on Crohn's Disease (28), refiere una asociación del $15 \%$ en la EC ( $6 \%$ con CU y $9 \%$ con EC), cifras más altas que las referidas en éste estudio, pero similares a las que obtenemos en el área de Gijón (32). También se ha confirmado que existen más enfermos con $\mathrm{CU}$ entre familiares de $\mathrm{EC}$ que al revés. El estudio de Comes y cols. (33), realizado en el área de Caláis, parece dar mayor importancia a los factores ambientales, al descubrir una alta incidencia de parejas sexuales con
EIIC, no diagnosticados antes de la convivencia. No obstante, ninguno de estos datos de familias, han aportado datos concluyentes de que los patrones familiares sean determinados por factores genéticos en lugar de ambientales (15,22,34-37).

Fenotipo (Extensión). Hemos utilizado en este estudio para la localización de la EC la clasificación más antigua (4), que con leves variaciones, fue propuesta por Sachar en la conferencia de consenso de Roma 1991 (38). No utilizamos la clasificación del consenso de Viena de 1998 (20), dado que nuestro estudio ha finalizado antes de ésta fecha. Los resultados de extensión que publican los distintos autores, son muy variables, probablemente debido a los diferentes criterios empleados a la hora de tipificar a 
TABLA V

EIIC. RATIO DE MORTALIDAD ESTÁNDAR (RMS)

\begin{tabular}{lccccccc}
\hline & CU & $(\%)$ & $\mathrm{Cl}$ & $(\%)$ & Extensión & EC & $(\%)$ \\
\hline EIIC & 1018 & 48 & 10,09 & 10,27 & 4,67 & $3,47-6,17$ & 0,000 \\
CU & 565 & 35 & 10,09 & 5,70 & 6,14 & $4,28-8,54$ & 0,000 \\
EC & 415 & 11 & 10,09 & 4,18 & 2,63 & $1,31-4,71$ & 0,001 \\
CI & 38 & 2 & 10,09 & 2 & 1,00 & $12,1-361$ & 0,000 \\
\hline
\end{tabular}

M uertes Esperadas = población $x$ tasa referencia $/$ 1000. RM S = M uertes observadas / muertes esperadas.

EIIC: enfermedad inflamatoria intestinal crónica; CU: colitis ulcerosa; EC: enfermedad de Crohn; Cl: colitis indeterminada.

los enfermos, más que a diferencias geográficas. Por ello, parece de interés aplicar en el futuro los criterios consensuados por la Conferencia de Viena (20). Igualmente la extensión para la CU, se expresa con gran variabilidad en la literatura, los datos reflejados, son demasiado diversos para sacar conclusiones validas, basta como ejemplo las proporciones de proctitis que refieren oscilan entre el $8 \%$ y el $59 \%$, debido al mismo problema. $(6,7,12-15,17,20-25,28,34,39)$.

Cirugía. El número de cirugías necesarias para el control de la enfermedad es significativamente superior en la EC respecto a las otras dos entidades, habiendo sido intervenidos el 26,62\% de todos los enfermos, correspondiendo a la EC una frecuencia de $50,36 \%$. Esta elevada tasa en relación con otras publicaciones $(24,26)$, se debe a que hemos incluido cirugías menores, como drenaje de abscesos perianales. Hemos incluido todas las intervenciones quirúrgicas, dado que nuestro interés es dar una aproximación de la morbilidad de las dos enfermedades. Nuestras tasas de cirugía en la CU son similares a las publicadas por Cella Lanau y cols. (24) y menores que las referidas por otros autores $(23,40)$, destacando una disminución de necesidad quirúrgica en los últimos 4 años que en años precedentes, probablemente por un mejor manejo terapéutico de éstos pacientes.

Mortalidad. Observamos un aumento del riesgo de muerte en la EIIC sobre la población general, que ya destacábamos en una publicación anterior (41), con una significación estadística tanto para CU como para EC, superior en CU en relación con EC ( $\mathrm{p}=0,046)$, y sin relación con el sexo. La mayoría de los autores documentan estos mismos resultados (13,42-44); sin embargo Munkholm y otros investigadores difieren de estas conclusiones, además de referir menor mortalidad en los últimos años $(45,46)$, mientras que otros como Brostom y cols. (47), con una mortalidad del $4 \%$ no encuentran diferencia entre ambas entidades. Creemos que estas diferencias en los resultados pueden deberse al diferente planteamiento entre estudios poblacionales y hospitalarios que es más manifiesto en servicios quirúrgicos. En un estudio más reciente Delcÿo y cols. (48) refiere que la mortalidad de la CU ha venido disminuyendo durante los últimos 40 años, mientras que en la EC aumenta entre 1950 y la mitad de 1970 hasta alcanzar un nivel similar a la mortalidad de la $\mathrm{CU}$, siguiendo un curso paralelo desde entonces, sugiriendo estos autores que puede existir un factor de riesgo responsable compartido de la mortalidad de ambas enfermedades y un factor adicional, responsable de la expresión de la EC exclusivamente. De igual forma, Palli y cols. (49) en su estudio de 920 EIIC comunica para la CU una SRM de 0,6; IC 95\% =0,4 - 0,8), cifra exacta a la nuestra con un riesgo del $40 \%$ para la EC, infiriendo la existencia de dos modelos de mortalidad divergentes para los pacientes con CU y EC, que ellos explican por las diferencias en los hábitos de fumar y por una mayor severidad de la EC.

En conclusión, con este estudio que abarca una importante población de enfermos, pretendemos aportar nuestros resultados epidemiológicos a la Enfermedad Inflamatoria Intestinal Crónica. Insistimos en la necesidad de unificar y utilizar los mismos criterios para todos los estudios epidemiológicos. Observamos la necesidad de discernir en los estudios de agregación familiar entre una causa genética heredo-familiar y causas ambientales. También creemos que sería oportuno realizar estudios de mortalidad amplios y poblacionales en nuestro país.

\section{Bibliografía}

1. Evans JG, Acheson ED. An epidemiological study of ulcerative colitis and regional enteritis in the Oxford area. Gut 1965; 6: 311-24.

2. Price Ashley B. Overlap in the spectrum of non-specific inflammatory bowel disease@Colitis Indeterminate@. J Clinic Pathol 1978; 31: 56777.

3. Lennard-Jones JE. Regional enteritis (Crohn`s disease): Definition and Diagnosis. Skandia International Symposia: Regional Enteritis. Stockolm: Nordiska Bolchandelns Forlag 1970: 108-9.

4. Lennard-Jones JE. Classification of inflammatory bowel disease. Scand J Gastroenterol 1989; 24: 2-4.

5. Truelove SC, Witts C. Cortisone in Ulcerative Colitis. Br Med J 1955; 2: $1041-8$.

6. Saro Gismera C, Lacort Fernández M, Argüelles Fernández G, Antón

Magarzo J, García López R, Navascues C, et al. Incidencia y prevalencia de la enfermedad Inflamatoria Intestinal Crónica en Gijón. Asturias. Gastroenterol Hepatol 2000; 23: 322-7.

7. Hinojosa J, Primo J, Lledó S, López A, Roig JV, Fernández J. Incidencia de Enfermedad Inflamatoria Intestinal en Sagunto. Rev Esp Enf Ap Dig 1990; 78: 283-7.

8. Solá Lamoglia R, García-Pugués AM, Monés Xiol J, Badosa Gallart C, Badosa Gallart J, Casellas F, el al. Enfermedad Inflamatoria Crónica Intestinal en Cataluña (Barcelona y Gerona). Rev Esp Enf Ap Digest 1992; 81: 7-14.

9. Brullet E, Rue M, Monserrat A, Gil M, Malet A, Mas P, et al. Estudio epidemiologico descriptivo de la colitis ulcerosa en un hospital comunitario (1985-1989). Med Clin Barc 1991; 97: 45-9. 
10. Maté Jiménez J, Muñoz S, Vicent D, Pajares JM. Incidence and prevalence of ulcerative colitis and Crohn's disease in urban and rural areas of Spain from 1981 to 1988. J Clin Gastroenterol 1994; 18: 27-31.

11. Shivananda S, Lennard-Jones J, Logan R, Fear N, Price A, Carpenter L, et al. Incidence of Inflammatory Bowel Disease across Europe: is there a difference between North and South? Result of the European Collaborative Study on inflammatory bowel disease. Gut 1996; 39: 690-7.

12. Brullet E, Bonfill X, Urrutia G, Ruiz Ochoa V, Cueto M, Clofent J, et al. Estudio epidemiológico sobre la incidencia de la enfermedad inflamatoria intestinal en cuatro áreas españolas. Med Clin 1998; 110: 6516.

13. Gower Rousseau C, Salomez JL, Dupas JL, Marti R, Nuttens MC, Votte A, et al. Incidence of inflammatory bowel disease in northem France (1988-90). Gut 1994; 35: 1433-8.

14. Langholz E, Munkholm P, Nielsen $\mathrm{OH}$, Kreiner S, Binder V. Incidence and prevalence of ulcertive colitis in Copenhagen county from 1962 to 1987. Scand J Gastroenterol 1991; 26: 1247-56.

15. Latour P, Belaiche J, Louis E, Fontaine F, Deflandre J, Loly J, et al. Incidence of inflammatory bowel disease in the province of Liege (Belgium). La Societe de Gastroenterologie Liegeoise. Acta Gastroenterol Belg 1996; 59: 3-6.

16. Shivananda S, Peña AS, Nap M, Weterman IT, Mayberry JF, Ruitenberg EJ, et al. Epidemiology of Crohn's disease in regio Leiden, The Netherlands. A population based study from 1979-1983. Gastroenterology 1987; 93: 966-74.

17. Manousos ON, Koutroubakis I, Potamianos S, Roussomoustakaki M, Gourtsoyiannis N, Vlachonikolis IG. A prospective epidemiologic study of Crohn's disease in Heraklion, Crete. Incidence over a 5-year period. Scand J Gastroenterol 1996; 31: 599-603.

18. Tragnone A, Corrao G, Miglio F, Caprilli R, Lanfranchi GA, Gruppo Italiano per lo Studio del Colon e del Retto (GISC). Incidence of inflammatory bowel disease in Italy: a nationwide population-based study Int J Epidemiol 1996; 25: 1044-52.

19. Gower-Rousseau C, Grandbastien B, Cortot A, Colombel JF. Epidemiology of inflammatory bowel disease: is there a "Belgian-French exception?". Acta Gastroenterol Belg 1996; 59: 2.

20. Gasche C, Schölmerich J, Brynskov J, D'Haens G, Hanauer SB, Irvine EJ, Jewell DP, et al. A simple classification of Crohn's disease: Report of the Working Party for the World Congresses of Gastroenterology, Vienna 1998. Inflamm Bowel Dis 2000; 6 (1): 8-15.

21. Stewenius J, Adnerhill I, Ekelund G, Florén C-H, Fork F-T, Janzon L, et all.Ulcerative Colitis and Indeterminate Colitis in the City of Malmö, Sweden. A 25-Year incidence study. Scand J Gastroenterol 1995; 30: 38-43.

22. Radhakrishnan S, Zubaidi G, Daniel M, Sachdev GK, Mohan AN Ulcerative colitis in Oman. A prospective study of the incidence and disease pattern from 1987 to 1994. Digestion 1997; 58: 266-70.

23. Tysk C, Järnerot G. Ulcerative proctocolitis in Örebro, Sweden. A retrospective epidemiologic study, 1963-1987. Scand J Gastroenterol 1992; 27: 945-50.

24. Cella Lanau J, López Zaborras J, Gomollón García F, Sáinz Samitier R. Enfermedad Inflamatoria Intestinal en Aragón: un diagnostico cada vez más frecuente. Rev Esp Enf Ap Dig 1995; 87: 363-7.

25. Kildebo S, Nordgaard K, Aronse O, Breckan R, Buhol PG, Jorde R, et al. The incidence of ulcertive colitis in Northern Norway from 1983 to 1986. The Northern Norwegian Gastroenterology Society. Scand J Gastroenterol 1990; 25: 890-6.

26. Monferrer Guardiola R, Martín Jiménez JA, Pedraza Sanz RG, Moreno Sánchez I, Soler Bahillo E, Hinojosa del Val J. Incidencia de la Enfermedad inflamatoria intestinal en el Área de Salud 02 de Castellón (1992-1996). Rev Esp Enferm Dig 1999; 91: 33-9.

27. Pera A, Bellando P, Caldeiro D, Ponti V, Astegiano M, Barletti C, et al. Colonoscopy in inflammatory Bowel Disease. Gastroenterology 1987; 92: 181-5.

28. Spanish Epidemiological and Economic Study Group on Crohn's disease. Epidemiological and clinical features of Spanish patients. Europ J
Gastroenterol Hepatol 1999; 11: 1121-7.

29. Van Gossum A, Adler M, De Reuck M, Devis G, Fiasse R, Vanheurverzwijn R, et al. Epidemiology of inflammatory bowel disease in Brussels' area (1992-1993). Acta Gastroenterol Belg 1996; 59: 7-9.

30. Cottone M, Cipolla C, Rosselli M. Epidemiology and pathogenesis of Crohn's disease. Epidemiologia e patogenesi della malattia di Crohn.SO: Chir Ital 1995; 47 (5): 1-7.

31. Satsangi J, Parkes M, Jewell DP, Bell JI. Genetics of inflammatory bowel disease. Clin Sci (Colch) 1998; 94 (5): 473-8.

32. Saro Gismera C, Lacort Fernández M, Argüelles Fernández G, Antón Magarzo JL, Navascues CA, García López R. Epidemiología de la enfermedad inflamatoria intestinal crónica en Gijón. Asturias. Gastroenterol Hepatol 2001; 24 (5): 228-34.

33. Comes MC, Gower Rousseau C, Colombel JF, Belaïche J, Van Kruiningen HJ, Nuttens MC, et al. Inflammatory bowel disease in married couples: 10 cases in nord Pas de Calais region on France and Liege country of Belgium. Gut 1994; 35: 1316-8.

34. Tavarela Veloso F, Fraga J, Carvalho J. Inflammatory bowel disease in Oporto. A prospective hospital study. Scand J Gastroenterol 1989; 24: 32-5.

35. Meuci G, Vechii M, Torgano G, Arrigonia T, Prada A, Rocca F, et al. Fammiliar aggregation of inflammatory bowel disease in Northern Italy. A multicenter study. Gastroenterology 1992; 103: 504-19.

36. Bayless TM, Z. Tokayer A, Polito II J M, Quaskey SA, Mellits ED, Harris ML. Crohn's disease: Concordance for site and clinical type in affected family members- Potential hereditary influences. Gastroenterology 1996; 111: 573-9.

37. Monsén U, Bernell O, Johansson C, Hellers G. Prevalence of Inflammatory Bowel Disease among relatives of patients with Crohn's Disease. Scand J Gastroenterol 1991; 26: 302-6.

38. Sachar DB, Andrews HA, Farmer RG, et al. Proposed classification of patient subgroups in Crohn's Disease. Gastroenterol Int 1992; 5: 141-54.

39. Lennard-Jones JE, Shivananda S, and the RC-IBD Study Group. Clinical uniformity of inflammatory bowel disease at presentation and during the first year of disease in the North and the South of Europe. Eur J Gastroenterol-Hepatol 1997; 9: 353-9.

40. Hiwatashi N, Yao T, Watanabe H, Hosoda S, Kobayashi K, Saito T, et al. Long-term follow-up study of ulcerative colitis in Japan. Gastroenterology 1995; 30: 13-6.

41. Saro Gismera C, Lacort Fernández L, Argüelles Fernández G, Anton Magarzo JL, Suárez González A, García López R, et al. Mortalidad y causas de muerte en Enfermedad Inflamatoria Intestinal Crónica. Gijón. Asturias. Rev Esp Enferm Dig 1999; 91: 199-203.

42. Ekbom A, Helmick CG, Zack M, Holmberg L, Adami HO. Survival and causes of death in patients with inflammatory bowel disease: a population-based study. Gastroenterology 1992; 103: 954-60.

43. Persson PG, Bernel O, Leijonmarck CE, et al. Survival and cause-specific mortality in inflammatory bowel disease: a population-based cohort study. Gastroenterology 1996; 110: 1339-45.

44. Weterman IT, Biemond I, Peña AS. Mortality and causes of death in Crohn's disease. Review of 50 years experience in Leiden University Hospital. Gut 1990; 31: 1387-90.

45. Munkholm P, Langholz E, Davidsen M, Binder V. Intestinal cancer risk and mortality in patients with Crohn's disease. Gastroenterology 1993; 105: 1716-23.

46. Probert CS, Jayanthi V, Wicks AC, Mayberry JF. Mortality in patients with ulcerative colitis in Leicestershire, 1972-1989. An epidemiological study. Dig Dis Sci 1993; 38: 538-41.

47. Brostrom O, Monsen U, Nordenwall B, Sorstad J, Hellers G. Prognosis and mortality of ulcerative colitis in Stockholm Country, 1955-1979. Scand J Gastroenterol 1987; 22: 907-13.

48. Delcÿo F, Sonnenberg A. Commonalities in the time trends of Crohn's disease and ulcerative colitis. Am J Gastroenterol 1999; 94 (8): 2171-6.

59. Palli D, Trallori G, Saieva C, Tarantino O, Edili E, D'Albasio G, et al General and cancer specific mortality of a population based cohort of patients with inflammatory bowel disease: the Florence Study. Gut 1998; 42 (2): 175-9. 